\title{
Angioedema por uso de Inhibidores de la Enzima Convertidora de Angiotensina en Otorrinolaringología
}

\author{
Angioedema by users of Inhibitors of the \\ Angiotensin Converting Enzyme in Otorhynolaryngology
}

Francisco Tocornal $\mathrm{J}^{1}$, Tatiana Espinoza $\mathrm{B}^{1}$, Jan Karlsruher $\mathrm{S}^{2}$, Jimena Cevo $\mathrm{E}^{3}$.

\begin{abstract}
RESUMEN
Se presenta una revisión de los casos de angioedema asociado al uso de Inhibidores de la Enzima Convertidora de Angiotensina de nuestro hospital. $\mathrm{\theta}$ objetivo es dar a conocer esta patología a la comunidad médica. La edad promedio de presentación fue de 67,8 años. Los pacientes tomaron el medicamento por entre 1 y 96 meses, antes de la aparición de los síntomas. La cantidad de episodios de angioedema antes del diagnóstico fue entre 1 y 7 , el seguimiento después del tratamiento, entre 8 y 18 meses. $\theta$ síntoma más frecuente fue edema lingual, aunque una paciente requirió tratamiento en la Unidad de Cuidados Intensivos con intubación orotraqueal. Se presenta una revisión de la literatura, con énfasis en la epidemiología, diagnóstico, mecanismo fisiopatológico y tratamiento de la enfermedad.
\end{abstract}

Palabras clave: Angioedema, IECA, Hipertensión arterial.

\section{SUMMARY}

A review of the cases of angioedema associated to the use of inhibitors of the angiotensin in our hospital is presented. The objective is to make this pathology known to the medical community. The average age of presentation was at 67,8 years of age. The patients took the medicine for a period between 1 and 96 months, before the symptoms appeared. The amount of angioedema episodes before diagnosis was between one and seven, follow-up after treatment, between 8 and 18 months. The most frequent symptom was lingual edema, although one patient required treatment at the Intensive Care Unit with orotracheal intubation. A review of the literature is presented, with emphasis on the epidemiology, diagnostic, physiopathological mechanism and treatment of the disease.

Key words: Angioedema, IECA, arterial hypertension.

\footnotetext{
${ }^{1}$ Médico del Servicio de Otorrinolaringología, Hospital Naval Almirante Nef.

2 Jefe del Servicio de Otorrinolaringología, Hospital Naval Almirante Nef.

${ }^{3}$ Interna Escuela de Medicina, Pontificia Universidad Católica de Chile.
} 


\section{INTRODUCCIÓN}

日 angioedema, o edema angioneurótico se caracteriza por áreas de edema, generalmente asimétrico, induradas, bien demarcadas, frecuentemente eritematosas, no pruriginosas, sin descamación y de rápida instalación. Es provocado por un aumento de la permeabilidad vascular de las zonas afectadas. Característicamente es autolimitado ${ }^{1}$.

日 angioedema afecta principalmente áreas de la cabeza y el cuello, siendo la región oral (lengua y labios) el sitio más frecuente de presentación 2,3 que puede progresar a una obstrucción completa de la vía aérea y muerte por edema laríngeo ${ }^{4}$.

Los inhibidores de la enzima convertidora de angiotensina (IECA) son usados extensamente para el tratamiento de la hipertensión arterial crónica (HTA) y en diversas cardiopatías. Entre las complicaciones descritas para estos medicamentos están la tos (0\%-39\%) y el angioedema con una incidencia que varía entre $0,1 \%$ y $0,2 \% \%^{5-7}$ de los pacientes tratados ${ }^{1}$.

Esta reacción adversa suele aparecer al poco tiempo de uso del medicamento, pero puede ocurrir hasta varios años después de haber comenzado el tratamiento, y recurrir en forma intermitente mientras se mantiene el uso de IECA, sin un factor desencadenante preciso ${ }^{1}$.

日 enfrentamiento del paciente con angioedema exige tener en cuenta las posibles causas, los riesgos asociados y el tratamiento que debe ser llevado a cabo.

\section{OBJETIVO}

Se presenta un estudio descriptivo retrospectivo de nuestros casos de angioedema asociados al uso de IECA y una revisión de la literatura, que pretende difundir en nuestra comunidad científica el conocimiento de esta patología.

\section{MATERIAL Y MÉTODO}

Se revisaron las historias clínicas de los pacientes que consultaron por angioedema asociado al uso de IECA, en el Servicio de Otorrinolaringología de nuestro hospital entre octubre 2004 y noviembre 2005. Se obtuvieron datos de sexo, edad, tipo de IECA usado, tiempo de uso del fármaco antes del inicio de los síntomas, tiempo de uso del medicamento desde la aparición de los síntomas hasta que se diagnosticó la asociación con el IECA, localización del edema, número de eventos previo al diagnóstico, seguimiento en meses. $日$ seguimiento se completó con un llamado telefónico a los pacientes de los que no se disponía de datos completos en la historia clínica.

Se presenta una descripción de los casos evaluados.

\section{RESULTADOS}

Durante el período estudiado, se presentaron 5 casos de angioedema por uso de IECA, de los que se lograron obtener datos completos de todos los pacientes, excepto la dosis precisa de IECA en uno de ellos.

Los pacientes fueron 3 mujeres y 2 hombres, de edades entre 59 y 79 años, con un promedio de 67,8 años. Los 5 recibieron enalapril, en dosis desde $5 \mathrm{mg}$ al día, hasta $10 \mathrm{mg}$ cada 12 horas; el tiempo de uso del enalapril antes de la aparición de los síntomas fue desde 1 a 96 meses con un promedio de 32,2 meses. Todos los pacientes presentaron edema lingual, en 2 de ellos hubo además compromiso facial y labial y en un caso se presentó edema laríngeo. La paciente que presentó edema laríngeo, requirió una evaluación urgente por otorrinolaringólogo, que indicó su ingreso a la Unidad de auidados Intensivos con tubo orotraqueal por 24 horas con buena evolución (Fguras 1 y 2).

La cantidad de episodios de angioedema previo al diagnóstico fue entre 1 y 7 , en uno de los casos no se pudo determinar el número, porque el paciente lo describió como recurrente pero de poca intensidad, por lo que no había sido motivo de preocupación. $\mathrm{日}$ seguimiento de los pacientes fue de 8 a 18 meses con un promedio de 13,2 meses y en ninguno de los casos se volvió a presentar 


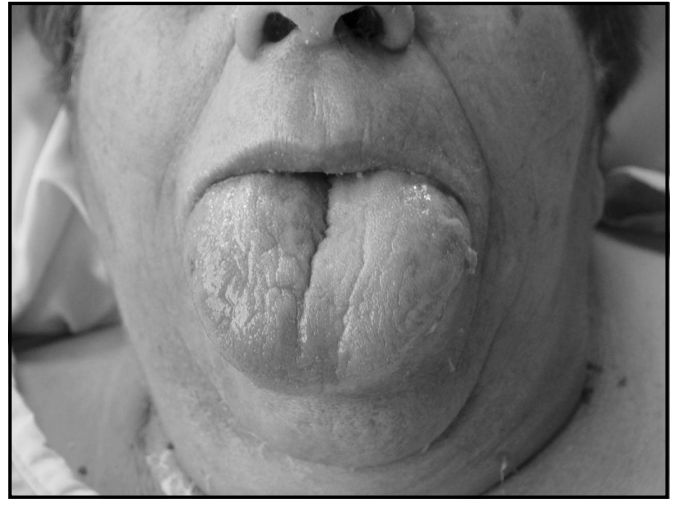

Figura 1. Angioedema pretratamiento.

angioedema luego de la indicación de suspender el enalapril. Llama la atención que en dos de los casos, constaba en la historia clínica en sus controles de hipertensión arterial crónica, la presencia de angioedema recurrente leve, sin haberse suspendido el medicamento.

Se presenta un resumen de los datos obtenidos en la Tabla 1.

\section{DISCUSIÓN}

Los IECA, son medicamentos ampliamente usados para el tratamiento de la HTA, insuficiencia cardiaca e insuficiencia renal crónica Su uso ha aumentado progresivamente. Sus efectos adversos más frecuentes son: hipotensión, disfunción renal, hipercalemia y

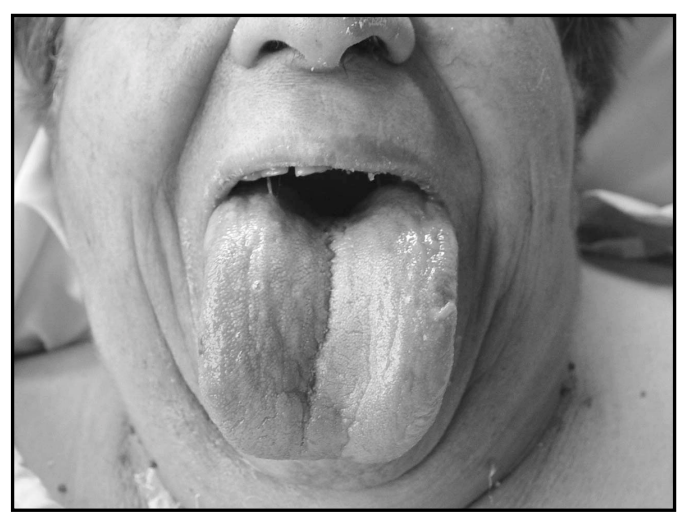

Figura 2. Angioedema postratamiento.

tos seca $(0 \%-39 \%)^{8}$. Pero existen otros que ocurren escasamente como hepatotoxicidad, rash, proteinuria, neutropenia y angioedema $(0,1 \%-0,2 \%$ de los pacientes tratados) ${ }^{5}$.

Se aceptan cuatro causas principales de angioedema ${ }^{10}$ :

- Hipersensibilidad alérgica tipo 1 dependiente de IgE, asociada a urticaria frecuentemente.

- Deficiencia congénita o adquirida del inhibidor C1 esterasa (angioedema congénito o asociado a neoplasias).

- Idiopáticas

- Drogas (no mediado por lgE: entre las que se encuentran: Ios IECA y los bloqueadores del receptor angiotensina II).

Los IECA, son fármacos que actúan en el sistema Renina-Angiotensina-Aldosterona. Inhiben

Tabla 1. Resumen de datos

\begin{tabular}{|c|c|c|c|c|c|c|c|c|}
\hline Caso & Sexo & $\begin{array}{l}\text { Edad } \\
\text { (años) }\end{array}$ & $\begin{array}{l}\text { Dosis } \\
\text { diaria de } \\
\text { enalapril } \\
\text { (mg) }\end{array}$ & $\begin{array}{l}\text { Tiempo de } \\
\text { consumo } \\
\text { (meses) previo } \\
\text { a síntomas }\end{array}$ & $\begin{array}{l}\text { Duración de } \\
\text { tratamiento } \\
\text { posterior al primer } \\
\text { episodio(meses) }\end{array}$ & Localización edema & $\begin{array}{l}\text { Número de } \\
\text { eventos previo } \\
\text { a suspensión } \\
\text { enalapril }\end{array}$ & $\begin{array}{l}\text { Seguimiento } \\
\text { (meses) } \\
\text { asintomático }\end{array}$ \\
\hline 1 & $M$ & 59 & 10 & 1 & 8 & Lengua (unilateral) & 7 & 18 \\
\hline 2 & $\mathrm{~F}$ & 68 & 5 & 24 & 12 & Lengua & recurrente & 17 \\
\hline 3 & $\mathrm{~F}$ & 79 & - & 24 & 0 & Lengua y laringe & 1 & 13 \\
\hline 4 & $\mathrm{~F}$ & 65 & 10 & 96 & 4 & Lengua, labio, párpado & 8 & 10 \\
\hline 5 & $M$ & 68 & 20 & 2 & 3 & Lengua, cara, labios & 3 & 8 \\
\hline
\end{tabular}


la conversión de angiotensina I en angiotensina II, y alteran la degradación de las bradicininas. La angiotensina II es un potente vasoconstrictor e interviene en la regulación de sodio y agua. Las bradicininas tienen efecto vasodilatador y aumentan la permeabilidad vascular. Por lo anterior estos medicamentos participan en la regulación de la presión arterial.

$\mathrm{B}$ angioedema producido por el uso de IECA, es un efecto secundario poco frecuente, comúnmente se presenta con edema del labio y lengua, en ausencia de urticaria y prurito generalizado. La gravedad puede variar desde escaso edema que regresa en forma espontánea, hasta una obstrucción de la vía aérea potencialmente mortal. Otros síntomas que pueden presentarse como consecuencia del edema son: disnea, tos, estridor, salivación excesiva, disfagia, y dolor abdominal ${ }^{1}$.

Se considera que $20 \%$ de los pacientes que presentan angioedema por IECA requerirán atención médica $\mathrm{日}$ tiempo de uso del medicamento pareciera no ser relevante, ya que el edema angioneurótico se ha visto que puede ocurrir desde horas hasta 2 años después de haber comenzado el tratamiento con IECA ${ }^{8}$. En nuestros pacientes, el promedio de tiempo de consumo de IECA previo a presentar el primer episodio de angioedema fue de 36 meses con una dispersión de 1 hasta 96 meses. La literatura menciona que es común que se presente cuando el tratamiento se inicia o, como máximo, hasta 1 semana después de haberlo comenzado ${ }^{1}$, aunque otros trabajos señalan que generalmente comienza años después y que recurre inconstantemente mientras éste se mantiene. En nuestros casos, los pacientes presentaron la complicación desde un mes hasta 8 años después de haber tomado regularmente el fármaco, por lo que vemos que la ausencia de síntomas en el primer período, no descarta este diagnóstico.

日 edema se revierte en horas después se suspender la droga y no parece ser dosis dependiente ${ }^{1}$, aunque nuestro paciente número 5 refería haber tomado $5 \mathrm{mg}$ de enalapril, día por medio, por muchos años, y fue dos meses después de ajustar su terapia a $10 \mathrm{mg}$ cada 12 horas que se presentó el angioedema. Puede ocurrir con dife- rentes IECA, pero en nuestros casos sólo tuvimos pacientes que tomaron enalapril, dado que es el IECA más usado en nuestro país.

No tuvimos casos de angioedema asociado a antagonistas del receptor de angiotensina II, aunque su relación con angioedema no está clara, se han reportado casos con menor frecuencia que con Ios IECA ${ }^{11}$.

Se ha reportado que los pacientes con angioedema idiopático tendrían mayor riesgo de desarrollar angioedema por el uso de IECA, por lo que en ellos, se desaconseja su uso ${ }^{1}$.

$\boxminus$ edema angioneurótico producido por otros tipos de drogas generalmente es mediado por IgE a través de mecanismos de hipersensibilidad tipo $\mathrm{I}^{10}$.

日 mecanismo a través del cual los IECA producen edema angioneurótico es desconocido, por lo que existen varias teorías de por qué producirían esta complicación. Se ha demostrado que la administración crónica de IECA, en especial Captopril ${ }^{\circledR}$, induce la formación de autoanticuerpos a tejidos específicos y anticuerpos antinucleares, por lo que se ha planteado, que el edema puede estar en relación a la formación de estos anticuerpos en personas genéticamente susceptibles ${ }^{1}$.

Por otra parte la enzima convertidora de angiotensina (ECA), degrada la bradicinina, por lo cual el uso de IECA produce un aumento de este péptido, de conocida acción vasodilatadora y de aumento de la permeabilidad vascular. En personas con edema angioneurótico hereditario, se pueden reproducir los síntomas con la infusión de bradicinina $^{1}$, no obstante se postula que este mecanismo no sería el único mediador del edema, ya que la administración crónica de IECA potencia la actividad sérica de la ECA, lo que no permitiría la acumulación de bradicinina'. De esta manera, se ha planteado la existencia de otro mecanismo, que sería la alteración del metabolismo de la bradicinina no mediado por ECA, como la carboxypeptidasa-N y la aminopeptidasa-P, que no son afectadas por los IECA ${ }^{12-14}$.

Se ha observado que el edema angioneurótico, puede ocurrir en forma espontánea en individuos que presentan deficiencia adquirida o hereditaria 
del inactivador de la esterasa del complemento, (C1-E), el que inhibe la fase inicial del sistema del complemento y la generación de quinina. Veinte a treinta por ciento de los pacientes con edema angioneurótico hereditario, tienen deficiencia funcional de este inactivador. Por lo que se cree que Ios IECA o sus metabolitos podrían causar su deficiencia a través de inactivar o inhibir su acción en aquellos individuos susceptibles ${ }^{1}$.

日 tratamiento del edema angioneurótico por IECA debe ser oportuno y en función de los síntomas del paciente, es importante la suspensión del fármaco y el manejo de la vía aérea.

En la consulta inicial, los pacientes deben ser examinados, en búsqueda de evidencia de compromiso respiratorio como estridor, edema lingual, disnea, disfagia y sialorrea.

En el manejo de la vía aérea se deben considerar la intubación traqueal $u$ otras medidas de control, como cricotiroidotomía o traqueostomía, según las condiciones del paciente y del centro donde se atiende al enfermo. Si el paciente está hemodinámicamente inestable puede ser necesario reposición de volumen y terapia vasopresora ${ }^{1-10}$. Si el diagnóstico no es claro, se puede considerar el tratamiento con antihistamínicos y corticoides si no hay contraindicación, aunque no está demostrada su utilidad en angioedema por IECA.

Las medidas de soporte se deben mantener hasta que el edema de la vía aérea haya sido resuelto. Los pacientes intubados debieran ser evaluados por un otorrinolaringólogo previo a su extubación.

Una vez resuelto el cuadro de edema angioneurótico se puede manejar al paciente en forma ambulatoria, cuidando reemplazar el tratamiento con IECA por otra terapia antihipertensiva. En la totalidad de nuestros casos la sustitución fue efectiva, aunque la literatura muestra casos de recidiva en pacientes en que la causa del angioedema era mixta o diferente de los IECA ${ }^{15}$.

\section{CONCLUSIÓN}

Los IECA juegan un rol muy importante en el tratamiento de la HTA e insuficiencia cardiaca. Con el aumento de su uso, se ha podido observar un mayor número de efectos secundarios y se espera un aumento en la frecuencia del edema angioneurótico. La consulta generalmente es referida al otorrinolaringólogo, y es por esto que para nuestra especialidad éste es un diagnóstico diferencial que se debe conocer.

Aunque la mayoría se presenta al inicio del tratamiento, esta complicación puede observarse hasta años después de iniciado éste.

En cualquier paciente que consulte por angioedema, especialmente si es recurrente, debe considerarse la posibilidad de angioedema por IECA y si está usando este tipo de medicamentos debe indicarse su suspensión.

\section{BIBLIOGRAFÍA}

1. ISRAILI ZH, HALL WD. Cough and angioneurotic edema associated with angiotensin-converting enzyme inhibitor therapy. A review of the literature and pathophysiology. Ann Intern Med 1992;117 (3): 234-42.

2. AGAH R, BANDI V, GUNTUPALLI KK. Angioedema: the role of ACE inhibitors and factors associated with poor clinical outcome. Intensive Care Med 1997; 23 (7): 793-6.

3. ZIRKLE M, BHATTACHARYYA N. Predictors of airway intervention in angioedema of the head and neck. Otolaryngol Head Neck Surg 2000; 123 (3): 240-5.

4. Dean DE, Schultz DL, Powers RH. Asphyxia due to angiotensin converting enzyme (ACE) inhibitor mediated angioedema of the tongue during the treatment of hypertensive heart disease. J Forensic Sci 2001; 46 (5): 1239-43.

5. VL田ING W, VAN AMSTERDAM JG, StRICKER BH, DE WILDT DJ. ACE inhibitor-induced angioedema; incidence, prevention and management. Drug Saf 1998; 18: 171-88.

6. WoOD SM, MaNn RD, RaWlins MD. Angiooedema and urticaria associated with angiotensin converting enzyme inhibitors. $\mathrm{Br}$ Med $\mathrm{J}$ (Cin Res Ed) 1987; 294 (6564): 91-2. 
7. Imman W, Rawsen N, Witon L et al. Postmarketing surveillance of enalapril I. Results of prescription event monitoring. BMJ 1998; 297, 826-9.

8. KYRMIZAKIS DE, PAPADAKIS CE, LIOLIOS AD, KARATZANIS AD, MALANDRAKIS S, SKOULAKIS CE, BIZAKIS JG, VEEGRAKIS GA. Angiotensin-converting enzyme inhibitors and angiotensin II receptor antagonists. Arch Otolaryngol Head Neck Surg 2004; 130 (12): 1416-9. Review.

9. PRACY JP, MOGLASHAN JA, WALSH RM, GEESON MJ. Angioedema secondary to angiotensinconverting enzyme inhibitors. $J$ Laryngol Otol 1994; 108 (8): 696-8.

10. MlyNaReK A, HaGR A, Kost K. Angiotensinconverting enzyme inhibitor-induced unilateral tonge angioedema Otolaryngol Head Neck Surg 2003; 129: 593-5.

11. DIONYSIOS E KYRMIZAKIS; OHARITON E PAPADAKIS; ANTONIOS D. LIOLIOS; ALEXANDROS D. KARATZANIS; STYLIANOS MALANDRAKIS; OHARALAMBOS E SKOULAKIS; JOHN G BIZAKIS; GEORGE A. VEEERAKIS. Angiotensin-Converting Enzyme Inhibitors and Angiotensin II Receptor Antagonists. Arch Otolaryngol Head Neck Surg 2004; 130: 1416-9.

12. Molinaro G, OGgNo M, Ṕ́RE M, LefaGe Y, Grivals N, AgOstoni A, AdAM A. Angiotensinconverting enzyme inhibitor-associated angioedema is characterized by a slower degradation of des-arginine(9)-bradykinin. J Pharmacol Exp Ther 2002; 303 (1): 232-7.

13. Adam A, ougno $M$, molinaro G, Perez $M$, LepaGE Y, AGostoni A. Aminopeptidase $P$ in individuals with a history of angio-oedema on ACEinhibitors. Lancet 2002; 359 (9323): 20889.

14. Letivire J, MuRPHEY L, HaRtirt T, SHAN RJ, SIMmonsW, Brown N. Dipeptidyl Peptidase IV Activity in Patients With ACE-Inhibitor-Associated Angioedema. Hypertension 2002; 39: 4604.

15. GCARDI M, ZINGALE LC, BgrgamaschinI L, AgOSTONI A. Angioedema associated with angiotensin-converting enzyme inhibitor use: outcome after switching to a different treatment. Arch Intern Med 2004; 164 (8): 910-3. 